\title{
Prevalencia y factores de riesgo relacionados con sangrado en la terapia con warfarina de los pacientes que ingresan al HUHMP de Neiva en el año 2011
}

\author{
Prevalence and risk factors related to bleeding of \\ patients when undergoing warfarina therapy at \\ Hernando Moncaleano Perdomo Hospital in \\ Neiva in 2011
}

Juan Diego Bonilla Hernández ${ }^{1}$, María Alejandra Leal Cardoso ${ }^{1}$, Leidy Tatiana López Artunduaga ${ }^{1}$, Lithey Cristina Poveda Conde ${ }^{1}$, Giovanni Caviedes Pérez ${ }^{2}$
Palavras-chave: Anticoagulación, sangrado, warfarina, INR.

\begin{abstract}
Resumen
El objetivo de la investigación fue determinar la prevalencia de sangrado como complicación por el uso de Walfarina y factores de riesgo asociados en pacientes mayores de 18 años que ingresan al Hospital universitario de Neiva Hernando Moncaleano Perdomo (HUHMP de Neiva). Es un estudio de corte transversal periodo retrospectivo del año 2011, con 67 pacientes del HUHMP que presentaban prolongación del INR (INR>3) y consumo de Warfarina, diagnóstico de sobreanticoagulación y /o presencia de sangrado con consumo de wararina. El análisis de datos se realizó con epi Info v.7.0. y Microsoft Office Excel 14.0.0 . Se encontro una prevalencia de sangrado asociada a la anticoagulación durante el 2011 fue del 59,70\%, siendo el sangrado mayor $(62,50 \%)$ el sangrado más frecuente.

Los principales diagnósticos con indicación para anticoagulación fueron: Prótesis valvular mecánica $(31,34 \%)$, Tromboembolismo venoso $(29,85 \%)$ y Fibrilación Auricular (16,42\%), no hubo relación entre el tipo de indicación y la presentación de sangrado. El 75,12\% de los pacientes tenían interacción medicamentosa principalmente con Lovastatina $(35,82 \%)$, Omeprazol $(28,36 \%)$ y Acetaminofén $(25,37 \%)$.

Hubo 4 muertes $(5,97 \%)$ durante el estudio, 2 (5\%) sangrados fatal. Los factores estadísticamente significativos fueron el control adecuado, posible factor protector para la presentación de sangrado, y ser mujer, posible factor de riesgo para presentar sangrado mayor. Como conclusion eExiste una alta prevalencia de sangrado entre los pacientes anticoagulados, el estudio sugiere como posible factor de riesgo (3 veces superior) ante sangrado mayor pertenecer al género femenino y como posible factor protector ser hombre y mantener un control adecuado, además de que puede presentarse sangrado independientemente del INR.
\end{abstract}

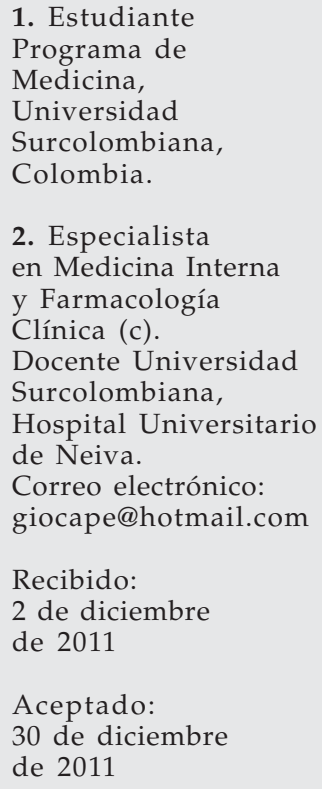

2. Especialista en Medicina Interna y Farmacología Clínica (c).

Docente Universidad Surcolombiana,

Hospital Universitario de Neiva.

Correo electrónico: giocape@hotmail.com

Recibido:

2 de diciembre

de 2011

Aceptado:

30 de diciembre

de 2011 
Key words: anticoagulation, bleeding, Warfarina, INR.

\begin{abstract}
This research aims at determining bleeding prevalence as Walfarina use complication and related risk factors in adult patients at Hernando Moncaleano Perdomo University Hospital in Neiva. (HMPUH). It is a retrospective period cross-sectional study from 2011, carried out with 67 patients from HMPUH, showing a INR prolongation (INR $>3$ ) and Warfarina intake, overanticoagulation diagnose and/or bleeding presence when taking Warfarina. Data analysis was carried out with epi Info v.7.0. and Microsoft Office Excel 14.0.0. A bleeding prevalence related to anticoagulation was found during 2011. It was of $59.70 \%$, being the highest bleeding $(62,50 \%)$, the most frequent.

Main diagnoses having indication of anticoagulation were: mechanical valve prostheses $(31,34 \%)$, venous thromboembolism $(29,85 \%)$, and auricular fibrillation $(16,42 \%)$. No relation was found between kind of indication and bleeding presence. $75,12 \%$ of patients had drug interaction mainly with Lovastatina (35,82\%), Omeprazol (28,36\%) and Acetaminophen $(25,37 \%)$.

There were 4 deaths $(5,97 \%)$ during the study, 2 (5\%) fatal bleeding. Statistically significant factors were adequate control, possible protective factor for bleeding presence and being woman, possible risk factor for showing major bleeding. In conclusion, there is high prevalence of bleeding among anticoagulated patients. This study suggests as possible risk factor (3 times higher) in the presence of major bleeding, belonging to female gender and as possible protective factor, being man and keep adequate control; besides bleeding may take place no matter INR.
\end{abstract}

\section{Introducción}

La Warfarina es un fármaco anticoagulante que actúa inhibiendo los factores de coagulación dependientes de vitamina $\mathrm{K}$ (Factores II, VII, IX y X y las proteínas anticoagulantes $\mathrm{C}$ y $\mathrm{S}$ ). El grado de depresión que ejerce sobre estos factores depende de múltiples factores como: dosis del fármaco administrada, interacción con otros fármacos, alimentos, comorbilidades, entre otros.

Existen muchas fuentes que plantean la dificultad del manejo de la Warfarina y su incidencia como uno de los fármacos con mayor número de eventos adversos reportados. El sistema de Reporte de Eventos Adversos de la FDA indicó que la Warfarina fue uno de los 10 medicamentos con mayor número de eventos serios reportados entre los años 1990 y 2000. En 1997, la Warfarina junto con la Insulina fueron identificados como los medicamentos más implicados en eventos adversos, según los datos Naciona- les de Servicios de Urgencias ${ }^{(1)}$ de los Estados de Unidos de América en el año 2002(2) y 2004 al $2005^{(3)}$.

Entre los eventos adversos más mencionados, el de mayor incidencia y conocimiento es el sangrado; los resultados encontrados sobre la frecuencia y los tipos de sangrado que se presentan son muy variados, debido a que en los estudios existen variaciones que van desde la definición de sangrado y de hemorragias graves, protocolos de tratamiento, estandarización del INR, tiempo de seguimiento, etc.

La incidencia anual de hemorragia mayor, ha variado entre 1,2 y 7 episodios por cada 100 pacientes en diferentes estudios de cohortes y de 0,5 a 4.2 en diferentes ensayos clínicos; y la incidencia de hemorragia menor varía entre 2 y 24 casos por cada 100 pacientes ${ }^{(4)}$. En otros estudios la incidencia de sangrado es de $0 \%$ a $63 \%$, dependiendo de los tipos de estudios, las clasificaciones utilizadas para cada uno de ellos y las poblaciones estudiadas ${ }^{(5)}$. 
En cuanto a nuestra región existe una gran falencia de datos epidemiológicos acerca de los eventos adversos relacionados con el uso de éste cumarínico; a pesar de su alta incidencia de hemorragias y al creciente número de pacientes que requieren de tratamiento con Warfarina, por ello se cree oportuno evaluar si dichos sangrados tienen diferente severidad y o frecuencia en relación a sus factores de riesgo relacionados.

\section{Metodología}

Se realizó un estudio de descriptivo transversal tomando como muestra $(n=67)$ el total de pacientes que presentaban INR prolongado (INR $>3$ ) y consumo de Warfarina, diagnóstico de sobreanticoagulacin por Warfarina y/o presencia de sangrado con consumo de Warfarina. Los Criterios empleados fueron:

\section{Criterios de inclusión}

1. Pacientes mayores de edad.

2. Pacientes con INR prolongado proveniente del laboratorio del HUHMP.

3. Pacientes con diagnóstico de sobreanticoagulación por Warfarina.

4. Pacientes que ingresaron y/o presentaron sangrado en el HUHMP bajo tratamiento con Warfarina, independiente del INR.

\section{Criterios de exclusión}

1. Pacientes en tratamiento con otros fármacos o con etiología diferente al uso de Warfarina responsable del sangrado.

\section{Consideraciones éticas}

Teniendo en cuenta el tipo de estudio, no se requiere carta de consentimiento informado por cada paciente. Se solicita el permiso correspondiente a la institución responsable de las Historias Clínicas teniendo en cuenta que los datos de identificación, como nombre y apellidos, no serán registrados en las encuestas, ya que no son va- riables importantes para el desarrollo del estudio; de esta manera se brindara seguridad y privacidad a los pacientes, sin violar su derecho a la intimidad.

\section{Análisis estadístico}

Se diseñó una encuesta con todas las variables examinadas en el estudio y utilizando la información obtenida de los datos de las historias clínicas de los pacientes, se analizaron las variables con el programa estadístico epi Info v.7.0. y microsoft Excel 14.0.0 (2010).

\section{Resultados}

En este estudio se revisaron un total de 186 historias clínicas de pacientes del HUHMP de las cuales se obtuvo una muestra de 67 pacientes. A los datos obtenidos de la encuesta aplicada sobre las historias clínicas se les calcularon sus respectivas medidas estadísticas según la escala de medición a la que pertenecían (proporción, mediana, moda, promedio, desviación estándar) con un intervalo de confianza del 95\% (IC 95\%).

De los 67 pacientes el 50,76\% fueron mujeres con una mediana de edad de 56,5 y en Hombres de 57; la mayoría de pacientes $(46,27 \%)$ provenían de municipios del Huila diferentes a Neiva. El 73,13\% de las historias clínicas no contenían información sobre la ocupación de los pacientes y el 17,91\% fueron amas de casa. Los principales diagnósticos con indicación para anticoagulación fueron: Prótesis valvular mecánica $(31,34 \%)$, Tromboembolismo venoso (TEV, TVP; $29,85 \%)$ y Fibrilación Auricular (FA, 16,42\%), pero no se encontró relación entre el tipo de indicación y la presentación de sangrado (Tabla 1).

A pesar de la rigurosidad que debe seguirse en el tratamiento con Warfarina, un $13,43 \%$ de los pacientes registraron una regularidad inconstante del consumo de la dosis y un $32,84 \%$ mostraron no seguir un control adecuado. La prevalencia de sangrado asociada a la anticoagulación fue del 
Tabla1. Características de base de los sujetos

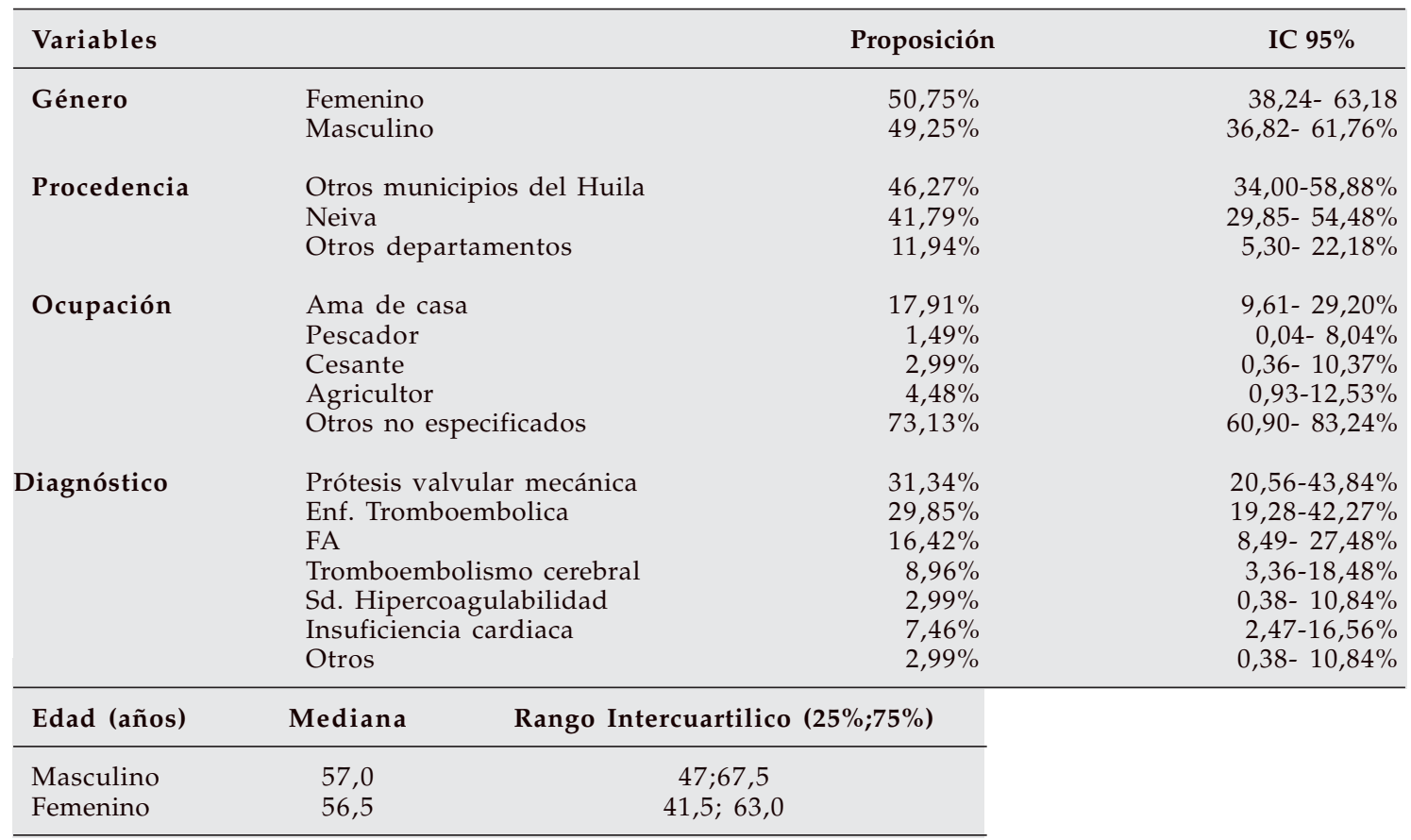

$59,70 \%$ y el tipo de sangrado más frecuente fue el sangrado mayor $(62,50 \%)$. La mortalidad relacionada con las alteraciones: INR prolongado y / o sangrado por Warfarina, fue de $5,97 \%$ (Tabla 2).
En los pacientes que además de warfarina consumían otros medicamentos (Tabla 3), se encontró que el 75,12\% tenían fármacos con interacción con la Warfarina, principalmente Lovastatina $(35,82 \%)$, Omeprazol

Tabla 2. Factores asociados a sangrado

\begin{tabular}{|c|c|c|c|}
\hline Variables & & Proporción & IC $95 \%$ \\
\hline \multirow{2}{*}{ Presentación de sangrado } & $\mathrm{Si}$ & $59,70 \%$ & $(47,00-71,51 \%)$ \\
\hline & No & $40,30 \%$ & $(28,49-53,00 \%)$ \\
\hline \multirow[t]{4}{*}{ Tipo de sangrado } & Mayor & $62,50 \%$ & $45,80-77,27 \%$ \\
\hline & Menor & $27,50 \%$ & $14,60-43,89 \%$ \\
\hline & Mortal & $5,00 \%$ & $0,61-16,92 \%$ \\
\hline & Fatal & $5,00 \%$ & $0,61-16,92 \%$ \\
\hline \multirow[t]{4}{*}{ Sitio de atención } & Urgencias & $49,25 \%$ & $(36,82-61,76 \%)$ \\
\hline & Piso & $32,84 \%$ & $(21,85-45,40 \%)$ \\
\hline & UCI & $13,43 \%$ & $(6,33-23-97 \%)$ \\
\hline & Consulta externa & $4,48 \%$ & $(0,93-12,53 \%)$ \\
\hline \multirow[t]{2}{*}{ Regularidad de las dosis } & SI & $86,57 \%$ & $(76,03-93,67 \%)$ \\
\hline & $\mathrm{NO}$ & $13,43 \%$ & $(6,33-23,97 \%)$ \\
\hline \multirow[t]{2}{*}{ Control adecuado } & SI & $67,16 \%$ & $54,60-78,15 \%$ \\
\hline & $\mathrm{NO}$ & $32,84 \%$ & $21,85-45,40 \%$ \\
\hline \multirow[t]{2}{*}{ Interacción con otros medicamentos } & SI & $76,12 \%$ & $64,14-85,69 \%$ \\
\hline & $\mathrm{NO}$ & $23,88 \%$ & $14,31-35,86 \%$ \\
\hline \multirow[t]{2}{*}{ Mortalidad } & SI & $5,97 \%$ & $1,65-14,59 \%$ \\
\hline & $\mathrm{NO}$ & $94,03 \%$ & $85,41-98,35 \%$ \\
\hline
\end{tabular}


Tabla 3. Medicamentos con mayor prescripción en pacientes con Warfarina $\left(^{*}=\right.$ Interacción con Warfarina)

\begin{tabular}{|c|c|c|}
\hline Medicamentos & Proporción & IC 95\% \\
\hline Metoprolol & $40,30 \%$ & $28,49-53,00 \%$ \\
\hline Enalapril & $38,81 \%$ & $27,14-51,50 \%$ \\
\hline Lovastatina* & $35,82 \%$ & $24,47-48,47 \%$ \\
\hline Omeprazol* & $28,36 \%$ & $18,01 \% \quad 40,69 \%$ \\
\hline Acetaminofén* & $25,37 \%$ & $15,53-37,49 \%$ \\
\hline Furosemida* & $20,90 \%$ & $11,92-32,57 \%$ \\
\hline Amlodipino & $13,43 \%$ & $6,33-23,97 \%$ \\
\hline Enoxaparina* & $13,43 \%$ & $6,33-23,97 \%$ \\
\hline Dipirona* & $10,45 \%$ & $4,30-20,35 \%$ \\
\hline Losartan & $10,45 \%$ & $4,30-20,35 \%$ \\
\hline Espironolactona* & $10,45 \%$ & $4,30-20,35 \%$ \\
\hline Amiodarona* & $11,94 \%$ & $5,30-22,18 \%$ \\
\hline Hidrolorotiazida & $7,46 \%$ & $2,47-16,56 \%$ \\
\hline Levotiroxina* & $5,97 \%$ & $1,65-14,59 \%$ \\
\hline Ranitidina & $7,46 \%$ & $2,47-16,56 \%$ \\
\hline Otros medicamentos con interacción & $46,27 \%$ & $34,00-58,88 \%$ \\
\hline
\end{tabular}

$(28,36 \%)$ y Acetaminofen (25,37\%); sin embargo, la relación entre interacciones medicamentosas como factor de riesgo para la presentación de sangrado mostro un OR de 0,4 con un IC $95 \%=(0,1-1,4)$ y una $p=0,08$ (Tabla 7).
El manejo terapéutico con mayor proporción $(71,64 \%)$ fue observación (Tabla 4), en la cual se ajustó o suspendio temporalmente la dosis de warfarina y fue la elección de manejo para los diferentes niveles de INR (Tabla 5) seguido por la administración de vitamina

Tabla 4. Tipo de manejo para INR prolongado o sangrado por Warfarina

\begin{tabular}{lcc}
\hline Tipo de manejo & Proporción & IC 95\% \\
\hline Observación & $71,64 \%$ & $59,31-81,99 \%$ \\
Vitamina K & $22,39 \%$ & $13,11-34,22 \%$ \\
Transfusión de plasma & $13,43 \%$ & $6,33 \% 23,97 \%$ \\
Transfusión de plasma y GR & $8,96 \%$ & $3,36-18,48 \%$ \\
\hline
\end{tabular}

Tabla 5. Tipo de manejo según los INR

\begin{tabular}{|c|c|c|}
\hline Tipo de manejo & Proporción & IC $95 \%$ \\
\hline \multicolumn{3}{|l|}{ INR 1-3 } \\
\hline Observación & $66,67 \%$ & $(38,38-88,18 \%)$ \\
\hline Vitamina $\mathrm{K}$ & $20 \%$ & $(51,91 \% 95,67 \%)$ \\
\hline Transfusión de plasma & $20 \%$ & $(4,33-48,09 \%)$ \\
\hline Transfusión de GR y plasma & $6,67 \%$ & $(0,17-31,95 \%)$ \\
\hline \multicolumn{3}{|l|}{ INR 3-5 } \\
\hline Observación & $90,91 \%$ & $(75,67-98,08 \%)$ \\
\hline Vitamina $\mathrm{K}$ & $9,09 \%$ & $(1,92-24,33 \%)$ \\
\hline Transfusión de plasma & $0,00 \%$ & Indefinido \\
\hline Transfusión de GR y plasma & $6,06 \%$ & $(0,74-20,23 \%)$ \\
\hline \multicolumn{3}{|l|}{ INR 5-9 } \\
\hline Observación & $50 \%$ & $(21,09-78,91 \%)$ \\
\hline Vitamina $\mathrm{K}$ & $41,67 \%$ & $(15,17-72,33 \%)$ \\
\hline Transfusión de plasma & $33,33 \%$ & $(9,92-65,11 \%)$ \\
\hline Transfusión de GR y plasma & $8,33 \%$ & $(0,21-38,48 \%)$ \\
\hline \multicolumn{3}{|l|}{ INR >9 } \\
\hline Observación & $40 \%$ & $(5,27-85,34 \%)$ \\
\hline Vitamina $\mathrm{K}$ & $40 \%$ & $(5,27-85,34 \%)$ \\
\hline Transfusión de plasma & $40 \%$ & $(5,27-85,34 \%)$ \\
\hline Transfusión de GR y plasma & 0 & Indefinido \\
\hline
\end{tabular}


$\mathrm{K}(22,39 \%)$, transfusión de plasma $(13,43 \%)$ y transfusión de plasma y Globulos rojos $(8,96 \%)$. Al establecer una relación entre las variables que podían estar asociadas al desarrollo de sangrado (Control adecuado, Regularidad de la dosis, Interacción, Género femenino y masculino) y la presentación de este, se encontró que la única variable estadísticamente significativa fue el control adecuado [OR 0,13 ; IC $95 \%=(0,03-0,53)$; $\mathrm{p}=0,0008$ ] y dado el valor de OR $<1$ podría asociarse a un factor protector para no presentar sangrado. (Tabla 6).

A pesar de que se esperaría una relación entre el INR y la presentación de sangrado (Tabla 7), no se encontró ninguna, dado a que se presentaron casos con INR de 1,06 con sangrado así como INR de 10,08 que no sangraron.

Los posibles factores de riesgo asociados al tipo de sangrado (Tabla 8, 9 y 10) se analizaron y se encontró que el control adecuado y el sexo masculino podrían ser factores protectores para presentar sangrado mayor (Tabla 9) con un OR=0,3, IC 95\%= $(0,1-0,8), p=0,015$ y OR de 0,32 , IC $95 \%=$ $(0,11-0,94), p=0,02$ respectivamente; para este tipo de sangrado también se encontró que ser mujer $(\mathrm{OR}=3,06, \mathrm{IC} 95 \%=1,05-8,91$, $\mathrm{p}=0,02)$ podría generar un riesgo indirecto 3 veces mayor que ser hombre de presentar sangrado.

Al realizar un análisis multivariado con regresión logística (Tabla 11) de los posibles

Tabla 6. Factores de riesgo asociados a la presentación de sangrado

\begin{tabular}{lccc}
\hline & OR & IC 95\% & P \\
\hline $\begin{array}{l}\text { Interacción con medicamentos } \\
\text { Control adecuado } \\
\text { Regularidad de la dosis }\end{array}$ & 0,4 & $(0,1-1,4)$ & 0,08 \\
\hline Género & 0,13 & $(0,03-0,53)$ & 0,0008 \\
$(0,16-3,1)$ & 0,33 \\
\hline F & 0,7 & IC 95\% & P \\
M & OR & $(0,54-3,88)$ & 0,23 \\
\hline
\end{tabular}

Tabla 7. Relación INR con presentación de sangrado

\begin{tabular}{lcccrrr}
\hline Presentó sangrado & Mínimo & $\mathbf{2 5 \%}$ & Mediana & $\mathbf{7 5 \%}$ & Máximo & Moda \\
\hline No & 1,3500 & 3,4400 & 3,8600 & 5,5300 & 10,8000 & 3,1500 \\
$\mathrm{Si}$ & 1,0600 & 1,8000 & 3,9300 & 5,1700 & 13,6000 & 1,1400 \\
\hline
\end{tabular}

Tabla 8. Factores de riesgo asociados al tipo de sangrado menor

\begin{tabular}{lccc}
\hline & A. Sangrado menor & & \\
\hline INR & OR & IC 95\% & P \\
\hline $1-3$ & 2,3 & $(0,5-9,4)$ & 0,13 \\
$3-5$ & 2 & $(0,5-7,6)$ & 0,16 \\
$5-9$ & 0 & Indefinido & 0,04 \\
$>9$ & 0 & Indefinido & 0,19 \\
\hline Interacción con medicamentos & 0,8 & $(0,18-0,34)$ & 0,38 \\
\hline Regularidad de la dosis & 0,64 & $(0,1-3,6)$ & 0,3 \\
\hline Control adecuado & 0,82 & $(0,21-3,1)$ & 0,39 \\
\hline Género & OR & IC 95\% & P \\
\hline Femenino & 0,42 & $0,11-1,63$ & 0,11 \\
Masculino & 2,33 & $0,61-8,89$ & 0,11 \\
\hline
\end{tabular}


Tabla 9. Factores de riesgo asociados al tipo de sangrado mayor

\begin{tabular}{lccc}
\hline & B. Sangrado mayor & & \\
\hline INR & OR & IC 95\% & P \\
\hline $1-3$ & 1,8 & 0,17 \\
$3-5$ & 0,36 & 0,02 \\
$5-9$ & 1,35 & 0,32 \\
$>9$ & 8,4 & $0,56-5-79)$ & 02 \\
\hline Interacción con medicamentos & 0,64 & $(0,37-4,8)$ & 0,23 \\
\hline Regularidad de la dosis & 0,65 & $(0,88-80,10)$ & 0,28 \\
\hline Control adecuado & 0,3 & $(0,15-2,72)$ & 0,015 \\
\hline Género & OR & $(0,1-0,8)$ & P \\
\hline Femenino & 3,06 & IC 95\% & 0,02 \\
Masculino & 0,32 & $1,05-8,91$ & 0,02 \\
\hline
\end{tabular}

Tabla 10. Factores de riesgo asociados al tipo de sangrado mortal

\begin{tabular}{lccc}
\hline & C. Sangrado mortal & & \\
\hline INR & OR & IC 95\% & P \\
\hline $1-3$ & 0 & Indefinido & 0,29 \\
$3-5$ & 1,03 & 0,19 \\
$5-9$ & 0 & Indefinido & 0,17 \\
$>9$ & 0 & Indefinido & 0,42 \\
\hline Interacción con medicamentos & 0,3 & $(0,01-5,0)$ & 0,23 \\
\hline Control adecuado & 0,47 & $(0,02-8,0)$ & 0,32 \\
\hline Género & OR & IC 95\% \\
\hline Femenino & 0,085 & $\mathbf{P}$ & 0,46 \\
Masculino & 1,16 & $0,05-14,30$ & 0,46 \\
\hline
\end{tabular}

Tabla 11. Análisis multivariado con regresión logística

\begin{tabular}{|c|c|c|c|c|c|c|c|c|c|}
\hline \multicolumn{2}{|l|}{ Variable } & \multicolumn{2}{|c|}{ Odds Ratio } & $95 \%$ & C.I. & Coefficient & S. E. & Z-Statistic & P-Value \\
\hline \multicolumn{2}{|c|}{ Control adecuado (Si/No) } & \multicolumn{2}{|c|}{0,17} & 0,03 & 0,79 & $-1,76$ & 0,78 & $-2,25$ & 0,0241 \\
\hline \multicolumn{2}{|c|}{ Regularidad de la dosis ( $\mathrm{Si} / \mathrm{No}$ ) } & \multicolumn{2}{|c|}{1,00} & 0,17 & 5,92 & 0,00 & 0,90 & 0,00 & 0,99 \\
\hline \multicolumn{2}{|c|}{ Interacción $(\mathrm{Si} / \mathrm{No})$} & \multicolumn{2}{|c|}{0,55} & 0,12 & 2,39 & $-0,59$ & 0,74 & $-0,79$ & 0,42 \\
\hline \multicolumn{2}{|c|}{ Femenino } & \multicolumn{2}{|c|}{1,41} & 0,46 & 4,31 & 0,34 & 0,56 & 0,60 & 0,54 \\
\hline \multicolumn{2}{|l|}{ INR3-5 } & \multicolumn{2}{|c|}{0,40} & 0,07 & 2,13 & $-0,89$ & 0,84 & $-1,06$ & 0,28 \\
\hline \multicolumn{2}{|l|}{ INR5-9 } & \multicolumn{2}{|c|}{0,23} & 0,03 & 1,46 & $-1,46$ & 0,94 & $-1,55$ & 0,12 \\
\hline \multicolumn{2}{|l|}{ INR>9 } & \multicolumn{2}{|c|}{1,35} & 0,08 & 20,88 & 0,30 & 1,39 & 0,21 & 0,82 \\
\hline Constante & & \multicolumn{2}{|c|}{ 1, $*$} & * & * & 2,70 & 1,22 & 2,20 & 0,02 \\
\hline \multicolumn{2}{|l|}{ Convergence: } & \multicolumn{3}{|c|}{ Converged } & & & & & \\
\hline \multicolumn{2}{|c|}{$\begin{array}{l}\text { Iterations: } \\
\text { Final }-2 * \text { Log-Likelihood: } \\
\text { Cases included: }\end{array}$} & \multicolumn{3}{|c|}{$\begin{array}{l}5 \\
74,8707 \\
67\end{array}$} & & & & & \\
\hline Test & Statistic & D.F. & $\mathbf{P}-\mathbf{V}$ & lue & & & & & \\
\hline Score & 13,5546 & 7 & & & & & & & \\
\hline Likelihood Ratio & 15,4725 & 7 & 0,0 & 304 & & & & & \\
\hline
\end{tabular}


factores de riesgo asociados a la presentación de sangrado la única variable estadísticamente significativa fue la de control adecuado \{OR:0,17; IC 95\% (0,03-0,79); $\mathrm{p}=0,02$ y al calcular la probabilidad $(\mathrm{P})$ de que los pacientes con un control adecuado presentaran sangrado esta fue tan solo de $0,002 \%$. Con el fin de poder establecer una asociación entre el control adecuado y la presentación de sangrado se calculó el $X^{2}$ esperado para una $\mathrm{p}<0,05$ con un grado de libertad de 1 , siendo el $\mathrm{X}^{2}$ esperado $=3,84$ menor que el encontrado $X^{2}=5,85$ por lo que podría plantearse finalmente que los pacientes que tienen un control adecuado tienen menor riesgo de presentar sangrado.

A la variable de control adecuado por ser estadísticamente significativa con un OR: 0,13; IC 95\% (0,03-0,53) y p 0,0008 se le cálculo:

- chicuadrado( $\left.x^{2}\right): 5,85$

degree free: 1

p value: 0,015

P para control adecuado $=0,002 \%$

Para esta variable con una $\mathrm{p}<0,05$ y un grado de libertad de 1 el valor de $x^{2}$ esperado es de 3,84.

\section{Discusión}

Está demostrado que la warfarina es uno de los fármacos más empleados tanto en nuestro país como en el resto del mundo en la práctica clínica, y a su vez de los que más presentan efectos adversos ${ }^{(6)}$, siendo el principal, el sangrado, sin embargo en nuestra región no existen datos sobre la prevalencia de sangrado. Las principales indicaciones para la terapia anticoagulante con warfarina fueron: prótesis de válvula mecánica $(31,34 \%)$, tromboembolismo venoso $(29,85 \%)$ y fibrilación auricular $(16,42 \%)$, indicaciones muy similares a otras descritas para la terapia de anticoagulación: TVP 30\%, Enfermedad valvular/prótesis $26 \%$, FA $14 \%{ }^{(7)}$.

En el presente estudio encontramos una prevalencia de sangrado debido a sobreanticoagulación del 59,70\% siendo el sangra- do mayor el más frecuente $(62,50 \%)$, probablemente esta prevalencia sea mayor debido al no reporte en algunas ocasiones en las historias clínicas. En cuanto al sangrado mayor existen reportes de estudios en los que la frecuencia de este evento varían entre $28,73 \%(1993)^{(7)}, 16 \%(2002)^{(8)}$ y $2,3 \%$ $(2005)^{(9)}$, todos estos inferiores a la frecuencia obtenida en nuestro estudio, posiblemente por el control inadecuado $(32,84 \%)$ y la falta de educación sobre los posibles eventos adversos de la terapia sumado a la falta de registro de eventos menores. Se encontró además que el control adecuado podría ser un factor protector (OR: 0,17; IC: 0,03$0,79, \mathrm{p}=0,02)$, resultado que concuerda con otros estudios.

Si se analiza a los pacientes por los valores de INR, independientemente de sus síntomas de consulta se encuentra que en los servicios de consulta externa entre 11 y $58 \%$ tienen niveles inadecuados mientras que en el servicio de urgencias el $72 \%$ están en esta condición, de estos últimos un $29 \%$ correspondieron a INR mayor de 3 y un $11 \%$ a un INR por encima de $5^{(10)}$, en nuestro servicio de urgencias encontramos que un $39,39 \%$ presentaron INR entre los rangos de 3 a 4,9 y un $19,7 \%$ mayor o igual a 5 , ambos datos 1,3 y 1,7 veces respectivamente, fueron superiores al estudio citado, alertando la necesidad de un cuidado más minucioso en los pacientes que reciben la terapia.

Aunque en la mayoría de los artículos se menciona ser hombre como un factor de riesgo para presentar sangrado(11), se encontró que una vez se presenta el sangrado las mujeres posiblemente tengan más riesgo de manifestar sangrado mayor (OR:3,06 IC:1,05-8,91 p:0,02). Los datos publicados en el presente artículo señalan que se puede presentar sangrado independiente del nivel del INR, datos que se relacionan con estudios en los que se han encontrado complicaciones inclusive dentro del rango establecido como deseado para la enfermedad de base ${ }^{(12)}$.

\section{Conclusiones}

- La prevalencia de sangrado asociada a la anticoagulación con Warfarina fue del 
$59,70 \%$ y el más frecuente fue el sangrado mayor $(62,50 \%)$.

- La mortalidad relacionada con las alteraciones: INR prolongado y/o sangrado por Warfarina, fue de $5,97 \%$.

- El sangrado puede ser independiente del INR.

- La mayoría de los pacientes que presenta sangrado y o sobreanticoagulación por Warfarina se hospitalizan en Urgencias.

- Ser mujer puede ser un factor de riesgo indirecto 3 veces mayor que ser hombre para presentar Sangrado Mayor, por ende el género masculino se asocia como factor protector.

- El Control adecuado puede asociarse como un factor protector, ya que la probabilidad de presentar sangrado en aquellos pacientes con un control adecuado fue tan solo de $0,002 \%$.

- La mayoría de pacientes (46.27\%) provienen de municipios del Huila de diferentes a Neiva.

- Las principales indicaciones para la terapia anticoagulante con warfarina fueron: prótesis de válvula mecánica $(31,34 \%)$, tromboembolismo venoso $(29,85 \%)$ y fibrilación auricular $(16,42 \%)$, indicaciones muy similares a otras descritas para la terapia de anticoagulación: TVP 30\%, Enfermedad valvular/prótesis $26 \%$, FA $14 \%(7)$.

\section{Agradecimientos}

A los pacientes encuestados, al Laboratorio Clínico, al Departamento de Epidemiologia y al personal de Archivo Clínico del Hospital Universitario Hernando Moncaleano Perdomo.

\section{Referencias}

1. HafnerJW,Belknap SM, SquillanteMD, BucheitKA. (200). Adverse drug eventsin emergency department. Ann Emerg Med, 39(3): 258-266.

2. Budnitz DS, Pollock DA, Mendelsohn AB, Weidenbach KN, McDonald AK, An- nest JL. (2005). Emergency department visits for outpatient adverse drug events: demonstration for a national surveillance system. Ann Emerg Med, 45(2):197-206.

3. Budnitz DS, Pollock DA, Weidenbach KN, Mendelsohn AB, Schroeder TJ, An nest JL. National surveillance of emergency department visits for outpatient adverse drug events. JAMA. 2006;296(15):1858-1866

4. Schulman S. (2003). Care of Patients receiving long-Term anticoagulant Therapy. $N$ Eng $J$ Med, 349:675-8.

5. McMahan DA, Smith DM, Carey MA, Zhou XH. (1998). The risk of major hemorrhage for outpatients treated with warfarin. J Gen Intern Med, 13:311-6

6. Tribiño G, Maldonado C, Segura O, Díaz J. (2006). Costos directos y aspectos clínicos de las reacciones adversas a medicamentos en pacientes hospitalizados en el servicio de medicina interna de una institución de tercer nivel de Bogotá. Biomédica, 26:31-41

7. Fihn SD, McDonell M, Martin D, et al. (1993). Risk factor for complications of chronic anticoagulation, a multicenter study; warfarin optimized out patient follow-up study group. Ann InternMed, 118:511-520.

8. Da Silva MS, Sobel M. (2002). Anticoagulants: to bleed or not to bleed, that is the question. SeminVascSurg, 15(4):256-267.

9. LINDH Jonatan D., HOLM Lennart, DAHL Marja-Liisa, ALFREDSSON Lars, RANE Anders. (2008). J ThrombThrombolysis, 25: 151-159, DOI 10.1007/s11239-007-0048-2.

10. Newman DH, Zhitomirsky I. (2006). The Prevalence of Nonthe- rapeutic and Dangerous International Normalized Ratios Among Patients Receiving Warfarin in the Emergency Department. Ann EmergMed, 48: 182-189.

11. Pineda Ruiz JC. (2009). Sobranticoagulación con Warfarina. Archivos de medicina, Manizales, 9(2):174-182.

12. Builes CE, Arango Cano WC. (2010). Frecuencia de sangrado en pacientes con enfermedades cardiovasculares anticoagulados con warfarina genérica vs. Coumadin. Acta Médica colombiana, 35(4):175-178. 